\title{
Philosophiques
}

\section{Le projet Épistémologique de Sartre}

\section{David Guy Joannis}

Volume 23, numéro 2, automne 1996

URI : https://id.erudit.org/iderudit/027398ar

DOI : https://doi.org/10.7202/027398ar

Aller au sommaire du numéro

Éditeur(s)

Société de philosophie du Québec

ISSN

0316-2923 (imprimé)

1492-1391 (numérique)

Découvrir la revue

Citer cet article

Joannis, D. G. (1996). Le projet Épistémologique de Sartre. Philosophiques, 23(2), 305-315. https://doi.org/10.7202/027398ar

\section{Résumé de l'article}

Contrairement à l'opinion répandue, l'échec de l'éthique et de l'ontologie ne constitue pas l'aboutissement de la pensée de Sartre, mais plutôt son point de départ. En effet, l'enjeu du projet de Sartre est le problème de la connaissance. d'utilisation que vous pouvez consulter en ligne.

https://apropos.erudit.org/fr/usagers/politique-dutilisation/ 


\title{
IE PROJET ÉPISTÉMOLOGIQUE DE SARIRE
}

\author{
PAR \\ DaVId GUY Joannis
}

\begin{abstract}
RÉSUMÉ : Contrairement à l'opinion répandue, l'échec de l'éthique et de l'ontologie ne constitue pas l'aboutissement de la pensée de Sartre, mais plutōt son point de départ. En effet, l'enjeu du projet de Sartre est le problème de la connaissance.

ABSTRACT: The failure of ethics and ontology. contrary to the common point of view, does not constitute the outcome of Sartre's philosophy but its starting point. Sartre's essential problematic is the problem of the foundation of knowledge.
\end{abstract}

Historiquement, la pensée philosophique de Sartre a été considérée sous différents angles qui, bien que présents et importants, n'apparaissent pas, du moins en tant que tels, suffisants pour rendre compte de la cohérence et de la profondeur de la démarche de Sartre ${ }^{1}$. Ces différentes interprétations sont les perspectives politique, ontologique et surtout éthique.

Ainsi, au sujet de la préoccupation politique, Merleau-Ponty et, surtout, Raymond Aron montrent ${ }^{2}$ le peu de souci qu'a Sartre pour l'analyse des problèmes politiques concrets et que le débat de Sartre vis-à-vis du marxisme se situe sur le plan strictement philosophique; aussi, ses conceptions politiques aboutissent-elles à une violence irrationnelle qui résulte d'une foi en la révolution, comme le lui fait admettre P. Verstraeten dans une importante entrevue ${ }^{3}$. Une telle foi étonne d'autant plus si l'on considère l'examen que Sartre fait de la question de la croyance : "Croire c'est savoir qu'on croit et

1. Nous voulons souligner que notre critique des interprétations traditionnelles de la pensée de Sartre n'est pas polémique et ne vise qu'à enrichir notre connaissance de sa pensée. C'est ainsi, du reste, qu'elle fut perçue par certains spécialistes de Sartre qui m'ont fait le privilège de lire le manuscrit de Sartre et le problème de la connaissance (Presses de l'Université Laval, automne 1996). Je rappellerais, sans prétention, que la philosophie de Hegel, ainsi que me l'a relaté $M$. Pierre Aubenque. fut prise au sérieux et enseignée en France aux lendemains seulement de la Seconde Guerre mondiale et fut l'objet d'une perception édulcorée et quelque peu romantique, à partir d'une lecture circonstancielle de la Phénoménologie de l'esprit qui ne représentait certes pas vraiment la pensée de Hegel.

2. M. Merleau-Ponty, Les aventures de la dialectique, Chapitre V : "Sartre et l'ultra-bolchevisme *, 1955, p. 142-295. Paris, Gallimard ; R. Aron, Marxismes imaginaires, D'une sainte famille à l'autre, p. 27-71. Paris. Idēes/Gallimard, 1970 ; R. Aron, Histoire et dialectique de la violence, Paris, Gallimard, 1973.

3. "Je ne suis plus réaliste ", Revue Gulliver, n 1, Paris, 1972, p. 39-46. 
savoir qu'on croit, c'est ne plus croire " puisque, dit Sartre, il s'agit alors d'une pure détermination subjective sans corrélatif extérieur.

Cette conception de la croyance, du reste, laisse entrevoir que la perspective ontologique ne peut non plus ètre retenue. Elle signifie qu'il n'y a pas de rapport entre un sujet et un objet, sinon sur un plan existentiel, c'est-à-dire pas sur le plan des concepts, y compris le concept de Dieu. En fait, la problématique ontologique de Sartre peut se résumer dans le problème de la totalité : pour Sartre, la quēte de la totalité est le drame de l'homme. En effet, la totalité n'est pas possible, puisque la conscience, ou pour-soi, ne peut ètre qu'en étant l'ètre qu'elle n'est pas et en n'étant pas l'être qu'elle est; la conscience est totalité détotalisée, c'est-à-dire elle est en tant que manque, puisqu'en cessant d'être manquant, elle cesse tout simplement d'être. La conscience est en n'étant pas l'ètre ; aussi, la totalité est impossible puisque la réalité humaine est engagée dans la totalité, et donc elle ne peut prendre un point de vue sur elle ou, si l'on veut, l'intériorité ne peut ètre saisie en tant qu'intériorité pour moi : "Ainsi, aucun point de vue sur la totalité n'est concevable : la totalité n'a pas de "dehors" et la question mème de son " envers " est dépourvue de signification ${ }^{5}$ ".

Jean Wahl, à propos de la possibilité d'une ontologie phénoménologique, déclare sans ambages qu'une ontologie de l'existence ne peut être qu'un échec ${ }^{6}$; la difficulté est de passer de l'existence à l'essence (et non l'inverse). L'examen de l'ontologie de Sartre, qualifiée par certains d'ontologie de l'échec ${ }^{7}$, montre qu'il faut plutôt y voir l'échec de l'ontologie elle-même. En effet, elle vise à constituer une ontologie en refusant l'être. Pour Sartre, il y a extranéité radicale de l'être au concept ; l'être n'est rien d'autre qu'un concept. À propos de la célèbre aporie du Ménon - il est impossible à un homme de chercher ce qu'il sait puisqu'alors il le sait, tout autant qu'il lui est impossible de chercher ce qu'il ne sait pas, puisqu'alors il ne sait pas quoi chercher - Kierkegaard affirme dans Les Miettes l'impossibilité de la connaissance; la réponse de Sartre à Ménon serait qu'il n'y a rien à chercher puisque l'essence est au bout de l'existence qui est pure liberté. Aussi, l'ontologie sartrienne apparait-elle comme une dissolution de l'ontologie.

Enfin, la dernière perspective, et non la moindre, est l'éthique ; elle constitue, en tout état de cause, le point de vue le plus répandu. La publication des Cahiers pour une morale l'a remise au premier plan, mème si, pour l'essentiel, le texte ne fait qu'approfondir des thèmes connus. Certes, la perspective est juste dans la mesure où l'agir, la vie en situations selon l'expression de Sartre, constitue le sujet d'un intérèt constant à travers toute son cuvre. Aussi, on peut qualifier Sartre de moraliste au sens d'une certaine tradition française ; son œuvre constitue une vaste "étude phénoménologique

4. L'Étre et le Néant (EN), Paris, NRF/Gallimard, 1943, p. 110 : Sartre reprend l'aporie de Les Possédés, de Dostoïevski : "Si Stavroguine croit, il ne croit pas qu'il croie. S'il ne croit pas, il ne croit pas qu'il ne croie pas. "

5. EN, p. 363.

6. J. Wahl, Essai sur le néant d'un problème, Paris, Deucalion, 1946.

7. F. Jeanson, Le problème moral et la pensée de Sartre, 1947, suivi de Un quidam nommé Sartre, Paris, Seuil, 1965, p. 142 ss. 
des attitudes humaines ${ }^{8}$ ". S. de Beauvoir rapporte que c'ètait là précisément le dessein de Sartre lorsqu'il écrivit les Cahiers. C'est ce qui ressort également des œuvres sur l'imaginaire, la question juive, Genet et Flaubert, ainsi que des analyses de $L^{\prime} E$ tre et le Néant, telle celle sur le regard, et évidemment de toute son œuvre romanesque et de théātre.

Les faits montrent que la conception morale de Sartre est surtout attribuable à Jeanson, à Beauvoir et à Gorz. Sur le fond, la perspective s'avère insuffisante en ce que la conception sartrienne de la conscience comme liberté absolue est incompatible et réfractaire à toute finalité, puisque la finalité est susceptible de compromettre ou d'amoindrir le caractère absolu de cette liberté et ce, même en instituant la liberté elle-même comme valeur. Or, le devoir-être de toute morale dissimule une finalité. Même Francis Jeanson, qui associe Sartre à la problématique morale, dans un texte ${ }^{9}$ commentant l'entrevue précitée avec Verstraeten, doit reconnaitre la position sans équivoque de Sartre sur ce sujet : Sartre refuse, dit-il, tout fondement à la finalité positive - la liberté doit demeurer libre par le haut et par le bas. Du reste, en outre qu'il faille considérer que l'éthique est vouée au mème sort que l'ontologie dont il dit qu'elle est indissociable ${ }^{10}$, la position de Sartre est clairement exprimée :

Ainsi toute Morale qui ne se donne pas explicitement comme impossible aujourd'hui contribue à la mystification et à l'aliénation des hommes. Le " problème " moral nait de ce que la Morale est pour nous tout en mème temps inévitable et impossible. Laction doit se donner ses normes èthiques dans ce climat d'indépassable impossibulitée ${ }^{11}$.

Autrement dit, par définition, l'homme aliéné ne peut accéder à une réalité qui lui est inaccessible, de la même manière que le sourd ne peut avoir l'idée de la hauteur du son. Aussi le constat de Sartre est-il double. D'une part, non seulement la morale est impossible aujourd'hui, mais elle est une mystification aliénante. D'autre part, si le problème moral est certes un fait inévitable, la morale néanmoins, quant à elle, demeure tout simplement impossible - d'une indépassable impossibilité. Le constat est ici explicite, mais il est une donnée claire du cheminement philosophique sartrien. Si l'on se refuse pourtant d'en tirer facilement la conclusion en ne considérant pas la philosophie de Sartre comme essentiellement une philosophie morale, sans doute est-ce en raison de la confusion résultant de ce

8. S. de Beauvoir, La cérémonie des adiewx, suivi de Entretiens auec J.-P. Sartre, aoūt-septembre 1974, p. 233-237.

9. F. Jeanson, Sartre dans sa vie, Paris, Seuil, p. 230, nota 1a, 1974.

10. F. Jeanson, voir la Préface de Sartre, 1947, p. 11-12.

11. J.-P. Sartre, Saint Genet, comédien et martyr, Paris, Gallimard, 1952, p. 212. Á ce mēme propos, Sartre, qui avait déjà noté l'impossibilité " dé tirer des impératifs de ces indicatifs " (En, p. 720 ss), réitère que " cette réflexion Ipure] je ne l'ai jamais décrite, j'ai dit qu'elle pourrait exister mais je n'ai montré que des faits de réflexion complice " "Sur L'Idiot de la famille ". SIT X, p. 104) ; ou encore, "L'Être et le Néant annonce une morale qui n'a jamais été donnée. (SIT X, p. 92). On trouve le même énoncé dans Vérité et existence : "...si la nature de l'homme est au bout de l'Histoire, l'inauthenticité doit être voulue pour elle-mēme comme condition même de la lutte historique. Toute doctrine de la conversion risque fort d'etre un ahistorisme. Toute doctrine de l'historicité risque fort d'ètre un amoralisme *. (p. 11). 
que les écrits de Sartre sont fortement imprégnès de préoccupations morales. Certes, ces préoccupations sont réelles; seulement, elles apparaissent à l'examen de la réalité vécue comme un problème, et non pas à titre de solutions qui seraient celles d'une philosophie morale.

Mais on ne peut faire fi ou diminuer la portée du constat sartrien quant à la morale et ne pas admettre que, à partir de là, sa réflexion philosophique se soit articulée autour de la recherche d'un fondement - d'où pourrait certes émerger une éthique. Ironiquement, il appert que cette recherche, à son tour, à travers une réflexion fondamentale sur le problème de la connaissance, aboutit aussi à un échec dū, quant à lui, à sa conception de la conscience. Bref, la problématique philosophique de Sartre est alors de définir les rapports entre une connaissance et une conscience, qui semblent s'exclure l'une l'autre. Mais rien n'étant fondé, il n'y a pas de morale non plus qui le soit ; l'impossibilité d'établir des normes reflète le vécu et les contradictions humaines. Plutōt que d'avoir une morale, peut-être n'y a-t-il, à ce jour, que des enseignements découlant d'une phénoménologie du vécu qui évoluent au fur et à mesure des expériences et des contextes, bref des situations?

Du reste, récemment, dans Les Temps modernes ${ }^{12}$, tant François Noudelmann, traitant de Sartre et de l'inhumain, que Hadi Rizk, parlant du livre Search for a Father de Robert Harvey, doivent faire le constat de l'impossibilité d'une morale sartrienne qui se situerait dans l'histoire et, par conséquent, de son échec à fixer une éthique. L'aliénation se fait le pendant de l'inauthenticité; elle n'a aucun critère pour se définir.

L'existence précède l'essence : la célèbre formule signifie que l'essence est uniquement l'affaire de la liberté. La passion de Sartre est de comprendre les hommes; elle n'a pas souci de la pensée normative, sinon pour la craindre et la refuser. Aussi, s'il est une issue sartrienne au problème de l'agir, ne se trouve-t-elle pas dans la formulation positive d'une morale, mais du côté de l'esthètique. Et pour Sartre, l'esthétique s'oppose à la morale ; on ne peut, considère$\mathrm{t}-\mathrm{il}{ }^{13}$, à la fois se situer sur le plan de la morale, c'est-à-dire sur le plan des conduites dans le réel par rapport au Bien, et sur le plan esthétique qui fait apparaitre l'irréel que nous admirons. La Beauté tue le désir - le réel n'est jamais beau, dit Sartre ${ }^{14}$. Il ne faut jamais oublier le vrai sens de la névrose du Salut par la littérature dont il fait la mise en scène dans Les Mots. Et surtout, il faut se rappeler que, pour Sartre, le démiurgique imaginaire est profondément apparenté $\dot{e}^{15}$ à l'existence d'une conscience qui est liberté.

12. Décermbre 1993.

13. L'Imaginaire, Paris, Idées/Gallimard, 1943, p. 371-379.

14. L'Imaginaire, p. 372.

15. L'Imaginaire, p. 361. Malgré sa propension pour l'art, Sartre est parfaitement conscient de " l'odyssée d'une conscience [... I à ne constituer qu'un monde irréel. Une conscience qui aurait perdu son ètre-dans-lemonde * (L'Imaginaire, p. 340) et de la nécessité d'un choix qu'impose l'art ou le monde imaginaire qui lui est imparti : "il faut donc que le pour-soi, dans son projet, choisisse d'être celui par qui le monde se dévoile, comme magique ou rationnel, c'est-à-dire qu'il doit, comme libre projet de soi, se donner l'existence magique ou l'existence rationnelle * (EN, p. 521). 
Certes, Sartre est fort conscient que l'esthétique se présente comme la négation de laction concrète et vécue qui détermine la problématique morale. Aussi son rapport à l'art ne peut-il ètre qu'ambigu. Dans Qu'est-ce que la littérature $?^{16}$, après avoir reconnu que morale et esthétique sont deux choses différentes let l'on sait son échec vis-à-vis de la morale), il affirme que c'est au fond de l'impératif esthétique que se trouve l'impératif moral, en ce que l'acte d'écrire se confond absolument avec la liberté elle-même tant par le fait de celui qui écrit et de celui pour qui il est écrit, que parce que cette liberté en constitue le seul sujet; dans un monde aliéné, l'art, entre autres par sa gratuité, sa générosité et son dépassement, apparait comme la véritable réalisation de la liberté, se faisant, dit-il, dévoilement de l'universel concret à l'universel concret. Bien sûr, Sartre doit finir par conclure qu'il s'agit d'une utopie, puisque l'écriture est d'abord un moment de réflexivité plutōt que d'action. Mais au-delà du discours formel, la conviction de Sartre parait différer; le savoir de l'art représente une action puissante et la seule certitude que nous ayons de cette liberté qui fonde la conscience et la connaissance. Ce n'est pas sans raison que toute la dernière partie des auvres majeures de Sartre, des Mots à L'Idiot, participe à une recherche esthétique, tant sur le fond que par la forme, comme si l'art prèsentait un lieu privilégié où se concilient conscience et connaissance ou, à tout le moins, qui nous permet d'en comprendre la relation. Enfin, quant à l'option de Sartre, la confession faite, vers la fin de sa vie, à Madeleine Chapsal est révélatrice: "Si j'étais Frantz, je ne me rongerais pas de remords : ... ètre dans une cellule et pouvoir écrire tranquillement ${ }^{17}$."

Bref, toute la démarche morale de Sartre, si tant est qu'on puisse lui conférer ainsi un sens qui ne soit pas sa négation, pourrait se résumer en la phrase qu'il prête à son héros dans l'Âge de raison: "je voudrais ne me tenir que de moi-même ${ }^{18}$ ".

Aussi, la lecture des écrits de Sartre fascine-t-elle et laisse perplexe. C'est que, outre les perspectives évoquées, il y apparaît, de façon omniprésente, une rationalité et une dialectique qui, contradictoirement, semblent se constituer comme la critique et le refus de la rationalité et de la dialectique - bref, une volonté de connaitre qui semble récuser la validité du savoir. Ce paradoxe est pourtant celui-là mēme auquel Sartre était confronté. Mais on n'a pas considéré vraiment la philosophie de Sartre sous l'angle du problème de la connaissance.

En effet, il faut voir que la philosophie de Sartre se constitue précisément à partir de l'échec de l'éthique. Un tel échec est conséquent à sa conception de la conscience comme liberté; si sa conception implique, comme il le clame dans un article clé sur la

16. Paris. Idées/Gallimard, 1948, p. 79 ss. C'est ce qui amène Sartre à conclure : "on n'écrit pas pour des esclaves" (p. 82). Voir aussi p. 196. Il n'est pas sans à propos de souligner que le véritable dessein de ce livre est de faire voir qu'on peut être et demeurer écrivain, tout en étant sympathisant marxiste; comme plus tard dans Les mots, il s'agit d'un réquisitoire masqué en faveur de l'art.

17. "Les écrivains en personne ", SIT IX, p. 27.

18. CEuvres romanesques, Paris, La Pléiade, p. 403. 
liberté cartésienne ${ }^{19}$, l'affirmation du règne humain par la liberté, se pose aussitōt le problème de la connaissance. Si conscience, liberté et existence sont tout, qu'en est-il de la connaissance qui, par définition, se pose comme n'étant pas l'existence ? Quel caractère apodictique présente-t-elle? Bref, comme dit Valéry dans L'âme et la danse, pourquoi connaitre et ne pas se suffire d'étre?

C'est là que se situe, à notre sens, le problème auquel Sartre est confronté et sur lequel il n'aura de cesse de travailler toute sa vie, ainsi qu'on peut brièvement le dégager de sa démarche. Dans ses premières œuvres ${ }^{20}$, Sartre dénonce l'insuffisance de la connaissance scientifique et conceptuelle. L'étude phénoménologique du rapport homme/chose met en cause la validité de la connaissance objective ; les faits qu'étudie la science reprêsentent une fausse connaissance et l'accumulation de faits, si grande soit-elle, n'arrive pas à représenter la réalitê humaine, les choses mémes selon l'expression de Husserl. L'objet n'est pas objectif ; l'objectivité n'est pas une propriêté de la chose, ni sa réalité comme elle se donne en personne. Sa critique est alors cinglante : "Il est aussi impossible d'atteindre l'essence en entassant les accidents que d'aboutir à l'unité en ajoutant indéfiniment des chiffres à la droite de $0,99^{21}$ ". Aussi, faut-il partir d'une toute nouvelle base épistémologique pour fonder la connaissance : il y a primauté de la conscience sur la connaissance - toute conscience est conscience d'elle-mème en même temps qu'elle est conscience d'un objet, selon la formule de Husserl.

Sartre s'applique aussitōt $\mathrm{t}^{22}$ à radicaliser la pensée de Husserl par la critique du Cogito cartésien. La démarche du doute méthodique est un artifice logique, puisque le doute présuppose ce dont il doute; Descartes a escamoté la donnée essentielle qui veut que la conscience qui dit Je pense n'est pas celle qui pense. De ce fait, le Cogito empoisonne ${ }^{23}$ la conscience et obnubile la véritable connaissance, qu'il prend un point de vue sur le réel, qui est hors du réel. Aussi, faut-il considérer qu'il n'y a pas de Je dans la conscience; la conscience n'a pas besoin d'ètre un objet pour exister, comme il en résulte du Je transcendant de l'acte réflexif. $\mathrm{Si}$ " exister et avoir conscience d'exister ne font qu'un" ", la conscience est autonome, sans contenu, et sans passivité (par opposition à l'inertie de la chose); il y a ainsi, selon une idée fondamentale de la philosophie de Sartre, priorité ontologique du

19. SIT I, p. 315-335.

20. L'Imagination, Paris, Guadrige/PUF, 1936 ; Esquisse d'une théorie des émotions, Paris, Hermann, 1963 (1939) : "Une idée fondamentale de Husserl : l'intentionnalité $"$. SIT I, etc.

21. Esquisse d'une théorie des émotions, p. 9-10. Sartre n'a alors cesse de dénoncer la perte de spontanéité, de naïveté qui rend la science peu adéquate à une connaissance réelle, La transcendance de l'Ego, p. 87-92.

22. "Conscience de soi et connaissance de soi ". Bulletin de la Société Française de philosophie, tome XLII, $\mathrm{n}^{\circ} 3$, avril-juin 1948, p. 49-71), La transcendance de l'Ego, Recherches Philosophiques, Paris, 1936-1937, p. 85-123. etc.

23. $\mathrm{TE}$, p. 99 : "La réflexion " empoisonne " le dèsir [...] Avant d'être "empoisonnés "mes désirs ont été purs ; c'est le point de vue que j'ai pris sur eux qui les a empoisonnès ".

24. L'Imagination, p. 125 ; aussi, L'Imaginaire, p. 311. 
réfléchi, soit un Cogito préréflexif pour lequel il n'y a pas dualité sujet/objet. La conscience est non-savoir radical.

Le problème de la connaissance devient, dès lors, l'enjeu de l'ontologie sartrienne. Certes, une analyse détaillée du texte complexe qu'est L'Étre et le Néant est nécessaire afin de faire ressortir les tenants et aboutissants de la problématique. De façon très succincte, elle se présente ainsi.

La conscience, ou si l'on veut le Cogito préréflexif, se détermine comme Négation; elle se détermine, dans un mouvement de surrection, à ne pas être l'être : "le pour-soi n'est jamais "25. Aussi, à la diffêrence de l'ètre inerte, opaque, fermé sur lui-mème, sans rapport et ne renseignant sur rien, la conscience peut-elle modifier son rapport à l'être ${ }^{26}$ - elle est liberté. Cet arrachement, qui l'oppose à la positivité de l'en-soi, signifie que la conscience ek-statique ne peut coïncider avec elle-même en tant qu'elle se détermine à exister en n'étant pas. Il en ressort qu'il est dirimant à la conscience de tenter d'être en n'étant pas son ne pas ètre ; elle ne peut être objet à elle-mème qu'en cessant précisément d'ètre la Négation qu'elle est.

S'il en est ainsi, qu'est-ce que la connaissance ? Et pourquoi la connaissance ? La réponse représente le point culminant de l'étude du pour-soi et constitue le cœur de l'ontologie. En fait, la connaissance quintessencie toute l'ontologie. Après avoir affirmé l'opposition irréductible de l'ètre et du non-ètre, il n'est pas possible à Sartre d'articuler une théorie de l'ètre qui rende compte de sa totalité, sans éviter ce qu'il considère ètre les pièges du réalisme et de l'idéalisme. À défaut de les réconcilier, Sartre doit néanmoins assurer un fondement à leur rapport, ce sans quoi sa propre réflexion n'a aucun sens. Pour ce faire, il ne faut pas, comme il en fait le reproche à Descartes ${ }^{27}$, dissocier les termes du rapport concret de l'homme au monde. La connaissance apparait alors comme ce fondement: elle est, en effet, relation, présence à, intuition, proximité. Qu'est-ce à dire, sinon que la connaissance se confond avec l'être ek-statique de la conscience?

Tout n'est cependant pas aussi simple. En effet, toute conscience n'est pas connaissance et, comme on l'a vu, la conscience qui se fait connaissance se démarque alors de la conscience qui est existence. L'ambiguïté résulte de ce que Sartre désigne sous le terme de connaissance une réalité ambivalente, estimant qu'elle présente deux facettes qui s'imbriquent l'une dans l'autre. Pour assurer le caractère apodictique des données de la réflexion, il importe, dit-il, que la réflexion ne soit pas conscience nouvelle, sans qu'il y ait pour autant une identité, ce qui annihilerait la connaissance. C'est pourquoi le terme connaissance doit signifier à la fois une connaissance pure et une connaissance impure, tout comme les notions de réflexion et de négation en comportent les deux aspects différents; bref, cette

25. EN, p. 196.

26. EN, p. 60 ss.

27. EN, p. 37 ss. Ce qui n'empêche pas Sartre d'affirmer que la rupture du lien concret d'être dissimule chez Descartes " bien avant le Heidegger de Von Wesem des Grundes, que l'unique fondement de l'être était la liberté ", SIT I, p. 334 . 
double réalité signifie à la fois le rapport en intériorité et le rapport en extériorité.

D'une part, il y a la connaissance pure; elle est le corollaire de la conscience comme Négation. Elle marque l'échec du pour-soi qui ne parvient pas à se fonder, en ce qu'il ne peut pas se confondre avec le ne pas être que la conscience a à être. La connaissance ne peut accéder à l'être : l'être nous est refusé, dit Sartre ${ }^{28}$. On comprendra mieux la démarche si l'on précise que Sartre décrit cette connaissance comme quasi-connaissance, intuition, présence, reconnaissance ; "Elle vise à faire exister le dévoilement d'un ètre qui est dévoilement dans son étre ${ }^{29}$ \%. Aussi cette connaissance est-elle idéale ${ }^{30}$ puisque l'objet qu'elle veut saisir est le réfléchi lui-même, lequel consiste luimême à ne pas être.

À cet égard, le rapport originel avec autrui manque l'exacerbation de cet échec de la connaissance. En effet, le rapport avec autrui n'est pas d'abord un rapport de connaissance, mais de reconnaissance, puisqu'il se passe au niveau du lien concret de l'existence. Autrui est une Négation qui nie ma Négation, cette fois, à la différence de l'ensoi, dans un rapport de réciprocité. La connaissance d'autrui n'est pas possible, non pas dans ce cas parce que la conscience est objet à elle-même, mais plutōt, inversement, parce que l'objet est extérieur au sujet ou, si l'on veut, "le récupéré n'est pas le récupérant ${ }^{31}$ ". Il n'en faut pas davantage pour conclure que l'objectivité n'a pas de sens. Cette connaissance n'est pas un savoir ; elle n'apprend rien.

Mais, d'autre part, il y a l'autre connaissance, que Sartre qualifie d'impure ou de complice, puisqu'elle fait comme si elle pouvait connaitre l'être en ne l'étant pas, comme si une saisie du dehors était possible. Elle est celle du discours, de l'analyse, de la déduction. Elle dit plus; bref, elle ne rend pas compte du réel, elle ne réfléchit plus. Comme des "plaques indicatrices ${ }^{32}$ "qui montrent la voie, le discours pointe l'objet, mais ne l'atteint pas; tel le miroir, il est l'illusion de la chose, même s'il dit tout sur elle. Il peut paraitre évident que cette connaissance dite en extériorité ne saurait réussir là où l'approche intériorisante n'a pas fonctionné 1 ll n'en est rien. La connaissance est paradoxe :

La réalité humaine se perd dans le monde par inauthenticité, mais êtredans-le-monde, c'est se perdre radicalement dans le monde par le dévoilement même qui fait qu'il y a un monde, d'ustensile en ustensilesans autre moyen que la révolution réflexive ${ }^{33}$.

Telle est là la seule issue à l'impasse de la connaissance qui est Négation ; c'est en tant que négation de la Négation, rupture d'être, que la connaissance prèsente un moyen d'accéder au réel. Bref, le déchiffrement du monde passe par les synthèses idéalisées de ce que Sartre nomme la révolution réflexive. La connaissance apparait alors

28. "Conscience de soi et connaissance de soi ", Bulletin de la Société française de philosophie, Tome XLII, $n^{\circ} 3$, avril-juin 1948, p. $48-91$ et plus précisément p. 79.

29. EN, p. 201.

30. Idem

31. EN, p. 361.

32. EN, p. 220.

33. EN, p. 251. 
comme la réalité singulière d'un acte par lequel la conscience en n'étant pas (son ne pas être) n'a pas d'autres moyens de ne pas ètre.

La connaissance, avec sa double dimension, marque ainsi un mouvement dynamique, progressif bien que non linéaire, à la manière d'une spirale : opposition infinie de la Négation sans cesse constituée et reconstituée de la conscience à l'être, négation de la Négation et, dans le cas d'autrui, négation de la Négation en tant que négation. Sartre répète cependant sans cesse qu'il n'y a pas de dialectique à partir de l'être. Il faut comprendre que, à cette époque, la conception que Sartre refuse est celle d'une dialectique associée à Hegel et au matérialisme dialectique. Néanmoins, la conscience sartrienne est dialectique. Après Guindey ${ }^{34}$, c'est ce que reconnaissent aussi Hartman, Catalano et Aronson, mais d'une autre manière, sans qu'ils en fassent ressortir les mouvements multiples et, surtout, sans qu'ils ne remarquent que cette dialectique est ètroitement associée au problème de la connaissance.

Cette dialectique, ètablie à partir de la conscience connaissante, devient explicite dans la Critique. Sartre dénonce alors la dialectique de la Nature pour lui opposer celle de la conscience. L'épistémologie matérialiste procède d'une insuffisance euristique; ses conclusions sont antidialectiques et irrationnelles puisque la Nature, comme l'ensoi, est inerte et passive. À travers la critique de la raison analytique, Sartre reprend le thème de la dénonciation de la science et $\mathrm{du}$ panconceptualisme : la raison ne doit pas se confondre avec la raison analytique - il faut désarrimer la raison du rationalisme. Bref, le savoir n'est pas conceptuel. Pour parvenir au véritable savoir, la méthode dite régressive-progressive et analytico-synthétique est euristique, puisqu'elle ne doit pas réduire ni escamoter les médiations multiples du concret : scruter le signifié et chercher le signifiant pour fixer la signification - l'uniforme se fait signifié parce que celui qui la porte se fait signifiant, ce sans quoi il est informe.

Il faut considérer, dit-il, que l'idéalisme de Hegel laisse échapper la matière et que le matérialisme de Marx nie la pensée. Pourtant, la Vérité n'est ni d'un côté ni de l'autre, pas davantage que dans la partie ou le tout, mais dans le mouvement interactif entre les deux ; la dialectique est dans le mouvement du rapport entre l'être et la connaissance. C'est sur le ton d'un quasi-manifeste, rappelant celui de son cèlèbre article sur l'intentionnalitè, qu'il affirme :

La seule théorie de la connaissance valable implique, comme en microphysique, que celle-ci soit pratique, c'est-à-dire qu'elle change le contenu ${ }^{35}$

34. G. Guindey, Le drame de la pensée dialectique, Hegel/Marx/Sartre. Paris, Vrin, 1976 ; K. Hartman, Sartre's Ontology -A Study of Being and Nothingness in the Light of Hegel Logic. Evanston, North Western University Press, 1966 ; R. Aronson, Sartre's Second Critic, Chicago, Chicago University Press, $1987 ; \mathrm{J}$. Catalano, A Commentary on Jean-Paul Sartre Critic of Dialectical Reason, Vol 1. Theory of Practical Ensembles. Chicago/London, Chicago University Press, 1986.

35. Questions de méthode (GM) dans Critique de la raison dialectique. Tome 1 (CRD) p. 30, note 1 . C'est ce que Sartre désigne comme la découverte capitale de l'expérience critique, c'est-à-dire de la connaissance, en tant qu'elle est praxis : "l'homme est médié par les choses dans la mesure même où les choses sont médiẻes par l'homme ", CRD, p. 165. 
À cela s'ajoute l'enseignement, d'ailleurs convergent, de Hegel à l'effet que la Vérité est totalisation, Sartre maintenant toutefois son refus qu'elle puisse être une totalité, la contradiction étant le moteur de l'histoire. L'êtude des rapports de groupe et de la société comme entité permet de réaffirmer les relations conflictuelles entre les individus et l'indépassable statut ontologique de la praxis individuelle $^{36}$. Il n'y a de réalité que l'homme.

Les conclusions de son épistèmologie, que Sartre qualifie de réaliste, rejoignent très précisément sa définition de la conscience, qui est ACTE, négativité dévoilante, projet en acte, connaissance située et totalisante : le PROJET est, dit Sartre, dépassement subjectif de l'objectivité vers l'objectivité, passage de l'objectif à l'objectif par l'intériorisation $^{37}$. La révolution réflexive est praxis reconstituante ${ }^{38}$. La conscience, on l'a vu, se définit comme une totalité détotalisée dont l'existence concrète n'est rien d'autre que la poursuite incessante d'une totalité, qui lui est par ailleurs forclose. Aussi, c'est par la retotalisation réflexive que la conscience définit, du moins provisoirement, son sens ou, si l'on veut, qu'elle en acquiert la compréhension, ne serait-ce que la connaissance de son incompréhension.

La re-totalisation réflexive assure l'intelligibilité et la rationalité de la totalisation constituée par la connaissance et l'action. Questionneur, questionné et question, dit Sartre, ne font qu'un. Par la connaissance située et dévoilante, la philosophie de la conscience fonde une vérité de l'homme et résorbe l'opposition entre l'existence et le savoir. La vérité, dit Kierkegaard, est l'acte de la liberté. La conception sartrienne de la conscience et de la liberté trouve aussi son fondement et sa cohérence dans la théorie de la connaissance.

Le problème de la connaissance est donc une articulation centrale de la pensée de Sartre. L'étude volumineuse de Sartre sur Flaubert "Vrai roman ${ }^{39}$ ", "roman vrai " ${ }^{40}$ ", présente la preuve démonstrative de sa théorie de la connaissance, la problématique expresse de Sartre etant alors "que peut-on savoir ${ }^{41}$ ? "... "comment puis-je étudier un homme $\mathrm{e}^{42}$. Outre son importance quant à la connaissance, et nous le notons en guise de conclusion, L'Idiot constitue une invitation pressante à considérer la pensée philosophique de Sartre du point de vue de l'esthètique. En effet, c'est une autre dimension essentielle à la compréhension de sa pensée, mais ignorée. Pourtant, la liberté démiurgique, la gratuité, l'Art et le jeu sont des signes qui annoncent l'importance de cette voie. Si Sartre avait fait un choix explicite de valeur, il eūt, sans doute, préférè le Beau au Bien. Sartre ne parle-t-il pas de connaissance jouie? Ce serait la suite logique à la présente étude; elle permettrait d'approfondir davantage et de situer plus fondamentalement encore la conception sartrienne de la connaissance.

36. Voir CRD, p. 567, 620 et 635.

37. QM. p. 66-67.

38. CRD, p. 139.

39. "Sartre par Sartre ", SIT IX, p. 123.

40. - Sur L'Idiot de la famille ", SIT X. p. 94.

41. L'Idiot de la famille. Tome 1, p. 7.

42. "Sartre par Sartre ", SIT IX, p. 114. 
On a l'habitude de dire que Sartre est le philosophe de son temps ; considérer Sartre comme n'étant qu'un personnage historique tend à en faire un philosophe méconnu et à nous faire oublier qu'il est un penseur profond affirmant, entre Nietzsche et Marx, cette vérité, à la fois lourde d'angoisse et pleine d'espérance : le monde est humain.

\section{Sillery}

